\title{
Front Matter: Volume 9626
}

, "Front Matter: Volume 9626," Proc. SPIE 9626, Optical Systems Design 2015: Optical Design and Engineering VI, 962601 (5 October 2015); doi: $10.1117 / 12.2218181$

SPIE. Event: SPIE Optical Systems Design, 2015, Jena, Germany 


\title{
PROCEEDINGS OF SPIE
}

\section{Optical Systems Design 2015: Optical Design and Engineering VI}

\author{
Laurent Mazuray \\ Rolf Wartmann \\ Andrew P. Wood \\ Editors
}

7-10 September 2015

Jena, Germany

Sponsored by

SPIE

Cooperating Organisations

European Optical Society (Finland)

Fraunhofer IOF (Germany)

Optonet (Germany)

Published by

SPIE 
The papers in this volume were part of the technical conference cited on the cover and title page. Papers were selected and subject to review by the editors and conference program committee. Some conference presentations may not be available for publication. Additional papers and presentation recordings may be available online in the SPIE Digital Library at SPIEDigitallibrary.org.

The papers reflect the work and thoughts of the authors and are published herein as submitted. The publisher is not responsible for the validity of the information or for any outcomes resulting from reliance thereon.

Please use the following format to cite material from these proceedings:

Author(s), "Title of Paper," in Optical Systems Design 2015: Optical Design and Engineering VI, edited by Laurent Mazuray, Rolf Wartmann, Andrew P. Wood, Proceedings of SPIE Vol. 9626 (SPIE, Bellingham, WA, 2015) Six-digit Article CID Number.

ISSN: 0277-786X

ISSN:1996-756X (electronic)

ISBN: 9781628418156

Published by

SPIE

P.O. Box 10, Bellingham, Washington 98227-0010 USA

Telephone +1 3606763290 (Pacific Time) · Fax +1 3606471445

SPIE.org

Copyright @ 2015 , Society of Photo-Optical Instrumentation Engineers.

Copying of material in this book for internal or personal use, or for the internal or personal use of specific clients, beyond the fair use provisions granted by the U.S. Copyright Law is authorized by SPIE subject to payment of copying fees. The Transactional Reporting Service base fee for this volume is $\$ 18.00$ per article (or portion thereof), which should be paid directly to the Copyright Clearance Center (CCC), 222 Rosewood Drive, Danvers, MA 01923. Payment may also be made electronically through CCC Online at copyright.com. Other copying for republication, resale, advertising or promotion, or any form of systematic or multiple reproduction of any material in this book is prohibited except with permission in writing from the publisher. The CCC fee code is 0277-786X/15/\$18.00.

Printed in the United States of America.

Publication of record for individual papers is online in the SPIE Digital Library.

\section{SPIE. DIGITAL}

SPIEDigitalLibrary.org

Paper Numbering: Proceedings of SPIE follow an e-First publication model. A unique citation identifier (CID) number is assigned to each article at the time of publication. Utilization of CIDs allows articles to be fully citable as soon as they are published online, and connects the same identifier to all online and print versions of the publication. SPIE uses a six-digit CID article numbering system structured as follows:

- The first four digits correspond to the SPIE volume number.

- The last two digits indicate publication order within the volume using a Base 36 numbering system employing both numerals and letters. These two-number sets start with 00, 01, 02, 03, 04, $05,06,07,08,09,0 A, 0 B \ldots$. OZ, followed by 10-1Z, 20-2Z, etc. The CID Number appears on each page of the manuscript. 


\title{
Contents
}

\section{Part One}

\author{
ix Authors \\ xiii Conference Committee
}

\section{PLENARY OPTICAL SYSTEMS DESIGN}

962604 Diffractive optical elements made from photonic metamaterials [9626-502]

\section{SESSION 1 THEORY AND MODELLING I}

962606 Frits Zernike and microlithography (Invited Paper) [9626-1]

962608 Direct design of a two-surface lens including an entrance pupil for imaging applications [9626-3]

962609 Efficient simulation of autofluorescence effects in microscopic lenses [9626-4]

$96260 \mathrm{~A}$ Tolerancing the impact of mid-spatial frequency surface errors of lenses on distortion and image homogeneity [9626-5]

\section{SESSION 2 THEORY AND MODELLING II}

$9626 \mathrm{OB}$ Predicting polarization performance of high-numerical aperture inspection lenses [9626-6]

9626 OC Non-sequential modeling of commercial dichroic beamsplitters using Zemax [9626-7]

$9626 \mathrm{OD}$ Array projector design for projection on arbitrarily curved surfaces [9626-8]

9626 OE Nano-optical concept design for light management [9626-9]

$96260 G$ An alternative approach to depth of field which avoids the blur circle and uses the pixel pitch [9626-11]

$9626 \mathrm{OH}$ Research of aberration properties and passive athermalization of optical systems for infrared region [9626-12]

9626 Ol Modeling, simulation, and analysis of birefringent effects in plastic optics [9626-13]

\section{SESSION $3 \quad$ THEORY AND MODELLING III}

9626 0J Reducible complexity in lens design (Invited Paper) [9626-14] 
9626 OK Limitations of using M-squared for input beam characterization in simulation software [9626-15]

$9626 \mathrm{OL}$ Partially coherent light with discontinuous surfaces using Wigner functions [9626-16]

$96260 \mathrm{M}$ Aberration modeling of thermo-optical effects applied to wavefront fine-tuning and thermal compensation of Sodern UV and LWIR optical systems [9626-17]

$9626 \mathrm{ON}$ Chromatic variation of aberration: the role of induced aberrations and raytrace direction [9626-18]

962600 Fast evaluation of surface sensitivity on ghost [9626-19]

\section{SESSION 4 COMPUTATIONAL IMAGING}

$96260 Q$ Computational imaging: the improved and the impossible (Invited Paper) [9626-21]

\section{SESSION 5 OPTICAL MATERIALS}

9626 OR Optical lead flint glasses: key material in optics since centuries and in future [9626-22]

9626 OS Characterization and measurement results of fluorescence in absorption optical filter glass [9626-23]

9626 OT Diffractive optics with high Bragg selectivity: volume holographic optical elements in Bayfol $^{\circledR}$ HX photopolymer film [9626-24]

\section{SESSION 6 FREEFORM OPTICS I}

9626 OU Overview of surface representations for freeform surfaces (Invited Paper) [9626-25]

$9626 \mathrm{OV}$ Optical design through optimization using freeform orthogonal polynomials for rectangular apertures [9626-26]

9626 OW Performance comparison of polynomial representations for optimizing optical freeform systems [9626-27]

9626 0X Investigation of TMA systems with different freeform surfaces [9626-28]

9626 OY Using the 3D-SMS for finding starting configurations in imaging systems with freeform surfaces [9626-29]

\section{SESSION 7 FREEFORM OPTICS II}

962610 Diffraction effects in laser beam shaping systems [9626-32]

962612 Describing freeform surfaces with orthogonal functions [9626-34]

iv 
962613 Optical tolerancing of structured mid-spatial frequency errors on free-form surfaces using anisotropic radial basis functions [9626-35]

962614 Wide field-of-view bifocal eyeglasses [9626-36]

962615 Alvarez lenses: theory and applications [9626-37]

\section{SESSION 8 DESIGN SOLUTIONS I}

962617 Zoom lens design for projection optics [9626-39]

962619 Lens auto-centering [9626-122]

9626 IA Wide-angle lens miniaturization through foveated imaging [9626-42]

9626 1B Dual-band infrared lenses design [9626-43]

9626 1C A study of modulation transfer function of digital image system via microscanning technique [9626-44]

\section{SESSION 9 DESIGN SOLUTIONS II}

9626 IE Lens-mount stability trade-off: a survey exemplified for DUV wafer inspection objectives (Invited Paper) [9626-45]

$9626 \mathrm{IF}$ The use of low departure aspheric surfaces in high quality wide angle lenses [9626-46]

962611 Hyper-hemispheric lens distortion model for 3D imaging SPAD-array-based applications [9626-49]

9626 iJ Handheld probes and galvanometer scanning for optical coherence tomography [9626-50]

9626 1K Electronic holographic device based on macro-pixel with local coherence [9626-51]

SESSION 10 SPECTROMETER DESIGN

9626 IL Spectrometry: photon sorting at the speed of light (Invited Paper) [9626-52]

9626 iN Fluorescence imaging spectrometer optical design [9626-55]

962610 ELOIS: an innovative spectrometer design using a free-form grating [9626-56] 


\section{Part Two}

\section{SESSION 11 LASER OPTICS I}

$96261 Q \quad$ Photon handling on femtosecond ultrafast beamlines (Invited Paper) [9626-58]

9626 IR Positioning of Littrow mounted gratings in pulse compressors [9626-59]

9626 is Optical design and performance of F-Theta lenses for high-power and high-precision applications [9626-60]

\section{SESSION 12 LASER OPTICS II}

$96261 \mathrm{U}$ Spaceborne lasers development for ALADIN instrument inside ESA Aeolus mission (Invited Paper) [9626-62]

9626 IV Modeling of optical aberrations due to thermal deformation using finite element analysis and ray-tracing [9626-63]

9626 IW Modular optical design for flexible beam shaping of a top-hat profile [9626-64]

$96261 X \quad$ A miniaturized laser illumination module [9626-65]

\section{SESSION 13 SPACE APPLICATIONS I}

9626 IY Opto-mechanical design of the MTG FCI spectral separation assembly [9626-67]

$96261 Z$ Ring-field TMA for PRISMA: theory, optical design, and performance measurements [9626-68]

\section{SESSION 14 SPACE APPLICATIONS II}

$962621 \quad$ Optical alignment of the SPICE EUV imaging spectrometer [9626-70]

962622 Recent developments in the design and verification of crystalline polarization scramblers for space applications [9626-72]

962623 Very high stability systems: LMJ target alignment system and MTG imager test setup [9626-73]

962624 Design and analysis of an active optics system for a 4-m telescope mirror combining hydraulic and pneumatic supports [9626-74] 
962625 Wavefront curvature sensing in a $2.5 \mathrm{~m}$ wide-field telescope: design, analysis, and implementation for real-time correction of telescope alignment [9626-75]

962626 VNIR, MWIR, and LWIR source assemblies for optical quality testing and spectro-radiometric calibration of earth observation satellites [9626-76]

962627 ATLID receiving spatial and spectral filtering units: design and associated performances [9626-77]

962628 3MI OPD optical design: concept and performances [9626-78]

962629 Image irradiance distribution in the $3 \mathrm{MI}$ wide field of view polarimeter [9626-79]

9626 2A ATLID beam steering mechanism and derived new piezoelectric based devices for optical applications [9626-80]

$96262 \mathrm{~B} \quad$ Impact of aberrations on the measurement of the solar diameter with the SODISM instrument [9626-81]

\section{POSTER SESSION}

9626 2C Lightweight design and finite element analysis of primary mirror for the space telescope [9626-82]

$96262 \mathrm{D}$ A tunable integrated system to simulate colder stellar radiation [9626-83]

$96262 \mathrm{E} \quad$ Progress on the prevention of stray light and diffraction effects on the Thai National Telescope [9626-85]

$96262 \mathrm{~F} \quad$ Optical and mechanical design and characterization of the new baffle for the 2.4-m Thai National Telescope [9626-86]

96262 High-resolution light-sheet microscopy: a simulation of an optical illumination system for oil immersion [9626-89]

9626 2J Opto-mechanical door locking system [9626-90]

$96262 \mathrm{~L} \quad$ Imaging spectroscopic reflectometer based on pellicle beamsplitter [9626-92]

$96262 \mathrm{~N} \quad$ Research and design for focusing device of secondary mirror [9626-94]

$962620 \quad$ Polymer hybrid materials for planar optronic systems [9626-95]

$96262 \mathrm{P} \quad$ Effects of satellite platform's vibrations on the image quality of a remote sensing payload: system level design and challenges [9626-96]

$96262 Q \quad$ Multi-wavelength laser system designed for material processing [9626-97] 
$96262 R \quad$ Maximum allowable low-frequency plafform vibrations in high resolution satellite missions: challenges and look-up figures [9626-98]

962625 Automation design of cemented doublet [9626-99]

$96262 \mathrm{~T}$ Influence of aspherical surfaces description on aberration correction [9626-100]

$96262 \mathrm{U}$ Analysis of ghost images in a compound prismatic combiner for head-up-displays [9626-101]

$96262 \mathrm{~W}$ Heat generation and thermo-mechanical effect modeling in longitudinally diode-pumped solid state lasers [9626-103]

$96262 Y$ Modeling of interferometer scheme for chirped fiber Bragg grating recording [9626-105]

962631 Parametric synthesis of optical systems composed of thin lenses by using the planeparallel plate aberration properties [9626-108]

962632 Analysis of the quality of the image formed by the zoom-lens optical system based on the use of MATLAB and ZEMAX [9626-109]

962633 Beam shaping of supercontinuum pulses [9626-110]

962634 Distortion definition and correction in off-axis systems [9626-1 11 ]

962637 Comparison of optical design methods of freeform surfaces for imaging applications [9626-114]

962638 Three-component zoom systems for transformation of Gaussian laser beams [9626-115]

962639 Miniature the size of integrated sphere by three-lens design to measure the total light flux of wafer-level LED [9626-116]

$96263 \mathrm{~A}$ Optimization model for UDWDM-PON deployment based on physical restrictions and asymmetric user's clustering [9626-117]

$96263 \mathrm{~B}$ Effects of temperature variations on the performance of a space imaging system baffle [9626-118]

$96263 G$ Analytical new method to generation of nonlinear optical pulses in wave guides and analysis of its stability [9626-125]

$96263 \mathrm{H} \quad$ Nonlinear and non-Hermitian optical systems applied to the development of filters and optical sensors [9626-126]

$962631 \quad$ Near-to-eye displays with embedded eye-tracking by bi-directional OLED microdisplay [9626-127]

9626 3J Optical design and stray light analysis for the JANUS camera of the JUICE space mission [9626-128] 


\section{Authors}

Numbers in the index correspond to the last two digits of the six-digit citation identifier (CID) article numbering system used in Proceedings of SPIE. The first four digits reflect the volume number. Base 36 numbering is employed for the last two digits and indicates the order of articles within the volume. Numbers start with 00, 01, 02, 03, 04, 05, 06, 07, 08, 09, 0A, 0B...0Z, followed by 10-1Z, 20-2Z, etc.

Achilles, Karin, OA

Adhikari, Achyut, 01

Afonyushkin, A. A., is

Agócs, Tibor, 37

Álvarez Cid, Marcos X., OC

Amaro de Faria Júnior, A. C., 3G, 3H

Anctil, Geneviève, 19

Andrae, P., OE

Archer, Julien, 27

Arévalo, Germán V., 3A

Asensio Campazas, Alberto, 0C

Asundi, Anand, 0

Babington, James, 15

Bakholdin, A. V., 1B, 2U

Barbero, Sergio, 14

Barbisan, Diego, 2D

Barillot, F., 2A

Barsotti, Stefano, 12

Bartoli, Alessandro, 28, 29

Battarel, D., OM

Battistelli, E., $1 \mathrm{~N}$

Baumgarten, Judith, 31

Beier, M., OU

Belli, Fabio, 28

Belly, C., 2A

Bening, Matthias, OB

Benítez, P., OV

Berlioz, Philippe, 27

Berner, A., ON

Bézy, Jean-Loup, 22

Biertümpfel, R., OS

Bociort, Florian, $0 \mathrm{~J}$

Boehme, Beate, 00

Bonato, Matteo, 2D

Bonhoff, Tobias, $1 \mathrm{~V}$

Bouazzam, Achmed, $1 \mathrm{E}$

Bourgain, F., 2A

Bove, Giuseppe, 28

Bradu, A., $1 \mathrm{~J}$

Bravetti, Paolo, $1 \mathrm{U}$

Brenner, Stephan, 3l

Brömel, A., OU, OW, OX

Brousse, Emmanuel, IY

Bruder, Friedrich-Karl, OT

Bruno, Umberto, 28, 29

Buisset, Christophe, 2E, 2F

Buljan, M., OV

Büsing, Lasse, $1 \mathrm{~V}$

Bustin, Nick, $0 Q$
Calamai, Luciano, 1Z, 28, 29

Caldwell, Martin, 21

Carel, Jean-Louis, $1 Y$

Carles, Guillem, $0 Q$

Caron, Jérôme, 22

Caruso, Alberto, 28

Cernat, R., $1 \mathrm{~J}$

Chen, Luan-Ying, 39

Chen, Shougian, $\mathrm{OQ}$

Chen, Xinwen, 2C

Cheng, Chen-Chin, 39

Chidangil, Santhosh, 2J

Chung, Kao-Der, 39

Claeyssen, F., 2A

Claudi, Riccardo, 2D

Cocola, Lorenzo, 2D

Compain, Eric, 23, 26

Coppo, P., 1N

Cortese, Maxime, 26

Cosentino, Alberto, $1 \mathrm{U}$

Côté, Patrice, 19

Cottenye, Justine, IY

Cremonese, Gabriele, 34, 3J

Čudek, V., 2 L

Da Deppo, Vania, 34

Dallaire, Xavier, 1A

Dalzell, Kristen E., IF

Dannberg, P., IX

Dantcaranov, Ruslan, 2T

Day, J. P. R., $1 \mathrm{~L}$

De Clerca, Coralie, 10

Debei, S., 3J

Delage, Laurent, 23

Della Corte, V., 3J

Demian, D., $1 \mathrm{~J}$

de Saint Seine, Diego, 27

Desnoyers, Nichola, 19

Dhillon, Vik, 2E

Dobre, G., $1 \mathrm{~J}$

Doering, D., 17

D'Ottavi, Alessandro, $1 \mathrm{U}$

Doucet, Michel, 19

Downing, James, $0 Q$

Dubroca, Guilhem, 22

Duerr, Fabian, 08

Duma, V.-F., $1 \mathrm{~J}$

Durieux, A., OM

Eccleston, Paul, 21

Ekimenkova, Alisa, 2 Y 
Engel, A., OS

Erbe, Torsten, $1 \mathrm{E}$

Erculiani, Marco S., 2D

Esslinger, Moritz, 09

Ezhova, Kseniia, 31, 32

Ezhova, Vasilisa, 31

Fäcke, Thomas, OT

Fahr, Stephan, OB, 1E

Falkner, Matthias, 04

Fang, Yi-Chin, 1C

Farisato, Giancarlo, 2D

Fasold, Stefan, 04

Fehse, Karsten, 3I

Fischer, Stephanie, OD

Flebus, Carlo, 24, 25

Fludra, Andrzej, 21

Formaro, Roberto, 12

Fossati, Enrico, 12

Frassetto, Fabio, 1Q

Freitag, H.-J., $1 X$

Freitag, J., $1 \mathrm{X}$

Friso, E., 3J

Fuchs, U., IW

Fuss, P., OM

Gabrieli, Riccardo, 28, 29

Gaillac, Stephanie, 26

Garshin, Aleksei, 1B, 2T

Gauvin, Jonny, 19

Gavaud, Eric, 26

Giro, Enrico, 2D

Glastre, Wilfried, 26

Gloesener, Pierre, 10, 24, 25

Gorny, S. G., 1S

Grabovickic, D., OV

Greenway, Paul, 21

Greggio, D., 3J

Griffin, Doug, 21

Grimm, V. A., 1S

Grishina, N. Y., $2 U$

Gross, Herbert, 09, 0L, ON, OU, OW, OX, OY, 10

Guenther, M. M., 10

Hagen, Rainer, OT

Haghshenas, Javad, 2P, 2R, 3B

Hahn, Joonku, $1 \mathrm{~K}$

Hanft, M., 17

Hardy, Liam K., 2E

Harmann, David, $1 Y$

Hartmann, Peter, OR

Hartung, J., OU

Harvey, Andrew R., $O Q$

Hasenauer, David M., OK

Heintzmann, Rainer, 21

Hélière, Arnaud, 27

Hild, Olaf, 3l

Hincapié, Roberto C., 3A

Hintz, M., $1 X$

Hoffmann, H., 3J

Hönel, Dennis, OT

Hou, Zhe, OJ
Huang, Guo-Yi, $1 \mathrm{C}$

Hutiu, Gh., $1 J$

Irawati, Puji, 2E

Ivanova, Tatiana, 2S, 2T

Jamoye, Jean-François, 10

Jaumann, R., 3J

Jonas, Reginald P., IF

Joncour, Marc, IY

Kalinchenko, Galina, IR

Kim, Hwi, 1K

Kirschstein, S., OX

Körner, Martin, 20

Korotkova, Natalia, 25

Kramer, Daniel, 1R

Kulmon, P., 38

Kunc, Thierry, 23

Kwon, Jaebeom, $1 \mathrm{~K}$

Labate, Demetrio, 28, 29

Lajunen, Hanna, 33

Lakhdari, Fouad, 2W

Lamontagne, Frédéric, 19

Lanternier, Catherine, 23

Lara, L. M., 3J

Laver-Solelhac, Maxime, 23

Leblay, Pierrick, 26, 27

Leckngam, Apichat, 2E

Leischner, Ulrich, 2 I

Lépine, Thierry, 2E, 2F

Lerer, Alexander, IR

Li, Weiyan, 2C, 2N

Lin, Po-Ming, $1 \mathrm{C}$

Lippmann, Uwe, OW, OY, 10

Liu, Jiayao, OV

Liv, Yangyang, 2C, $2 \mathrm{~N}$

Loiseaux, Didier, 22

Loosen, Peter, IV

Lousberg, Gregory P., 24, 25

Lu, Xiang, 2

Lv, Qunbo, 2C, 2N

Ma, C., OW

Magrin, D., 3J

Maiorano, Michele, 28, 29

Makhlouf, Houssine, $1 Y$

Maksimovic, Milan, 13

Mangalgiri, G., OE

Manley, P., OE

Manolis, Ilias, 28, 29

Maquet, Philippe, 23, 26

Marque, Julien, 23, 26

Martaud, E., OM

Meftah, Mustapha, 2B

Meneghini, Matteo, 2D

Menzel, Christoph, 04

Metzner, Gerburg S., OY

Michaelis, H., 3J

Middleton, Kevin, 21

Mikš, A., 38

Milde, T., 17 
Milenky, Michael N., 2Q

Miñano, Juan C., OV

Miotti, Paolo, 1Q

Mohasel Afshari, Behzad, 3B

Möhl, A., IW

Moon, Woonchan, $1 \mathrm{~K}$

Moreau, Vincent, 10, 24, 25

Mouricaud, Daniel, $1 Y$

Munari, M., 3J

Muyo, Gonzalo, $0 Q$

Nádaský, P., 2L

Naletto, Giampiero, 34

Narasimhan, B., OV

Negrutiu, M. L., $1 \mathrm{~J}$

Nie, Yunfeng, 08

Nijkerk, M. D., $1 \mathrm{~L}$

Nikolic, Milena, OV

Nobis, T., ON

Notni, Gunther, OY

Novák, J., 38

Novák, P., 38

Ochse, Dennis, OA, OU, OW, 12

Ohlídal, M., 2L

Oleszko, M., OU, OW

Olivieri, Monica, 29

Orselli, Enrico, OT

Osmani, Ismahen, 2W

Oussalah, Meihdi, $1 Y$

Palumbo, P., 3J

Patil, Ajeetkumar, 2J

Patil, Saurabh Suhas, 2J

Pei, LinLin, 2N

Pereira Do Carmo, João, 27

Pernechele, Claudio, 11

Pertsch, Thomas, 04

Petruck, P., OX

Phillipon, Anne, 21

Piérard, Maxime, 24

Pirnay, Olivier, 25

Podoleanu, A. Gh., $1 \mathrm{~J}$

Pokorný, P., 38

Poletto, Luca, 1Q, 2D

Poshyajinda, Saran, 2E, 2F

Potheau, Hervé, 26

Pradal, Fabien, 2B

Prasit, Apirat, 2E, 2F

Prucker, Oswald, 20

Pyś, Grzegorz, $\mathrm{OH}$

Raevsky, Eugene V., $2 Q$

Ragazzoni, R., 3J

Reichel, S., OS

Reichmann, L., 12

Rewitz, Christian, OT

Richards, Tony, 21

Richert, Michaël, 22

Richichi, Andrea, 2E

Richter, Bernd, 3 l

Richter, Undine, OY

Riedel, Alf, OD
Riedel, W., OE

Riguet, François, 1Y, 2B

Rockstuhl, Carsten, 04

Rodenko, Olga, 09

Rodolfo, Jacques, IY

Rodrigues, Vanessa M., 2J

Rogers, Kevin, 21

Rolland, J. P., $1 \mathrm{~J}$

Rölle, Thomas, OT

Romanova, Galina E., $0 \mathrm{H}, 2 \mathrm{~S}, 2 \mathrm{~T}, 2 \mathrm{U}, 2 \mathrm{Y}$

Rubinstein, Jacob, 14

Rühe, Jürgen, 20

Rus, Bedrich, $1 R$

Saida, Tabet, 2W

Salasnich, Bernardo, 2D

Sandhya, V., $1 \mathrm{X}$

Saprykin, Dmitry L., $2 Q$

Satzer, Britta, OY

Sawangwit, Utane, 2E

Schädel, M., $1 X$

Schipani, P., 3J

Schmid, M., OE

Schmidt, S., 10

Schmitz, N., 3J

Schreiber, Peter, OD

Schühle, Udo, 21

Schumacher, Jean-Marc, 24

Schuster, Norbert, OG

Shafer, D., ON

Sieler, Marcel, OD

Sierra, Javier E., 3A

Simioni, Emanuele, 34

Sinescu, C., 1J

Singh, Manisha, 33

Somja, Aude, 24

Song, M., OE

Soonthornthum, Boonrucksar, 2E

Staude, Isabelle, 04

Steinkopf, R., OU

Stollenwerk, Jochen, IV

Stone, Bryan D., OK

Suetta, Enrico, $1 \mathrm{U}$

Sugranes, Pierre, 26

Taiti, A., IN

Tervo, Jani, 33

Thibault, Simon, $1 \mathrm{~A}$

Thienpont, Hugo, 08

Thompson, Kevin P., 1 Z

Thomschke, Michael, 31

Topala, F. I., 1 J

Tosh, Ian, 21

Tremblay, Mathieu, 19

Trivellin, Nicola, 2D

Tsakanikas, S., OE

Tsay, Ho-Lin, 1C

Tseng, Szu-Yuan, 1C

Tünnermann, Andreas, 09, 0X

Turunen, Jari, 33 
Uhlendorf, Kristina, OA, OB, 12

Vaché, Maxime, 27

Van Luen, Nguen, 32

Vasilyev, V. N., $2 U$

Villa, Federica A., 11

Vink, H. J. P., $1 \mathrm{~L}$

Vodák, J., 2L

Vogel, Uwe, 3l

Volatier, J.B. C.G., $1 \mathrm{~L}$

Voznesenskaya, Anna, 2Y

Vyhlidka, Stepan, IR

Wallace, Brian P., IF

Walther, Benny, 04

Walze, Günther, OT

Wang, JianWei, 2N

Wartenberg, Philipp, 3

Werschnik, Jan, OB, $1 \mathrm{E}$

Wickenhagen, S., IW

Williamson, David M., 06

Winzer, A. T., $1 \mathrm{X}$

Wood, Andy, OQ

Yin, G., OE

Yudin, K. V., is

Yurevich, V.I., 1S

Zhang, Dandan, 2C, 2N

Zhong, Minyi, OL

Zhong, Y., OU, OW, OX

Zhukova, Tatiana, 2T

Zuccaro Marchi, Alessandro, 10

Zusi, M., 3J

Zverev, Victor, 31, 32 


\title{
Conference Committee
}

\author{
Symposium Chair \\ Wilhelm Ulrich, Carl Zeiss AG (Germany) \\ Symposium Co-chairs \\ Juan Carlos Miñano, Universidad Politécnica de Madrid (Spain) \\ David M. Williamson, Nikon Research Corporation of America \\ (United States) \\ Honorary Symposium Chair
}

Tina E. Kidger, Kidger Optics Associates (United Kingdom)

Conference Chairs

Laurent Mazuray, Airbus Defence and Space (France)

Rolf Wartmann, Carl Zeiss Microscopy (Germany)

Andrew P. Wood, Qioptiq Ltd. (United Kingdom)

Conference Programme Committee

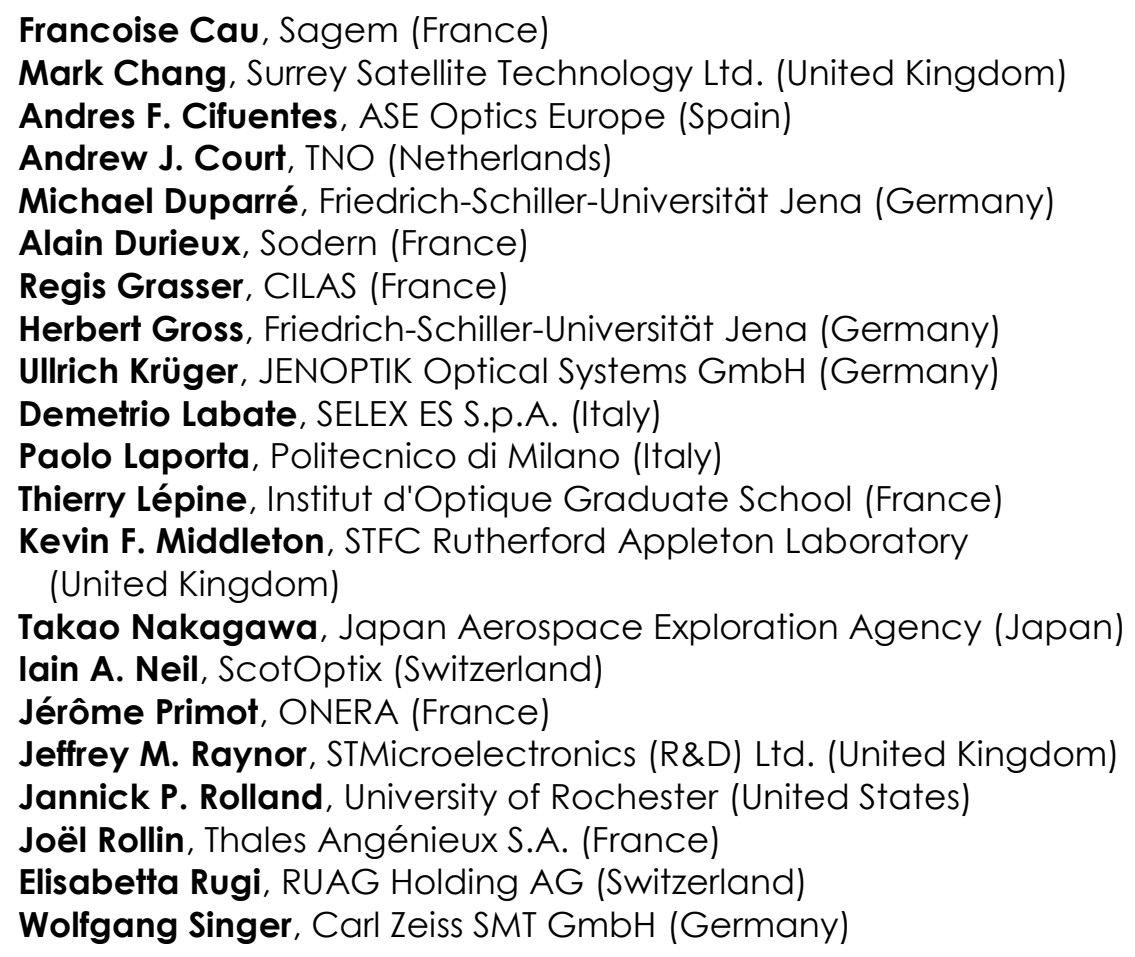


Simon Thibault, Université Laval (Canada)

Kevin P. Thompson, Synopsys, Inc. (United States)

Kristina Uhlendorf, JENOPTIK Optical Systems GmbH (Germany)

Min Wang, INO (Canada)

Richard N. Youngworth, Riyo-LLC (United States)

María J. Yzuel, Universidad Autònoma de Barcelona (Spain)

\section{Session Chairs}

1 Theory and Modelling I

Rolf Wartmann, Carl Zeiss Microscopy GmbH (Germany)

2 Theory and Modelling II

Andres F. Cifuentes, ASE Optics Europe (Spain)

3 Theory and Modelling III

James Babington, Qioptiq Ltd. (United Kingdom)

4 Computational Imaging

Francoise Cau, Sagem (France)

5 Optical Materials

Francoise Cau, Sagem (France)

6 Freeform Optics I

Ullrich Krüger, JENOPTIK Optical Systems GmbH (Germany)

7 Freeform Optics II

Herbert Gross, Friedrich-Schiller-Universität Jena (Germany)

8 Design Solutions I

Kristina Uhlendorf, JENOPTIK Optical Systems GmbH (Germany)

9 Design Solutions II

James Babington, Qioptiq Ltd. (United Kingdom)

10 Spectrometer Design

Laurent Mazuray, Airbus Defence and Space (France)

11 Laser Optics I

Rolf Wartmann, Carl Zeiss Microscopy GmbH (Germany)

12 Laser Optics II

Demetrio Labate, SELEX ES S.P.A. (Italy)

13 Space Applications I

Demetrio Labate, SELEX ES S.p.A. (Italy) 
14 Space Applications II

Kevin F. Middleton, STFC Rutherford Appleton Laboratory (United Kingdom)

15 Space Applications III

Laurent Mazuray, Airbus Defence and Space (France)

Proc. of SPIE Vol. 9626 962601-15

Downloaded From: https://www.spiedigitallibrary.org/conference-proceedings-of-spie on 26 Apr 2023 Terms of Use: https://www.spiedigitallibrary.org/terms-of-use 
Proc. of SPIE Vol. $9626962601-16$

Downloaded From: https://www.spiedigitallibrary.org/conference-proceedings-of-spie on 26 Apr 2023 Terms of Use: https://www.spiedigitallibrary.org/terms-of-use 